

\section{ELABORACIÓN DE UN PROGRAMA DE INVESTIGACIÓN CIENTÍFICA PARA JÓVENES DEL NMS QUE MOTIVE A SU PARTICIPACIÓN CIENTIIFICA}

○

\section{HEBER MIGUEL TORRES CORDERO}

\section{RESUMEN}

El crecimiento económico y la competitividad internacional de un país están íntimamente relacionados con su capacidad de desarrollo científico y tecnológico (Méndez, 2000). México es un país en donde la poca inversión en ciencia y un bajo índice en el nivel académico de la población, provocan falta de motivación entre los jóvenes para decidirse por carreras científicas e ingenierías y para dedicarse al desarrollo científico y tecnológico del país (Hernández, 2018). Pese a los esfuerzos mostrados por el Conacyt e instituciones privadas por realizar actividades enfocadas a la motivación científica de los jóvenes de bachillerato, son pocos los programas que se enfocan en la formación de jóvenes investigadores. En este trabajo se presenta el desarrollo de un programa de modalidad extracurricular a nivel de bachillerato cuyo objetivo es fomentar en estudiantes del Nivel Medio Superior el interés por la investigación y el desarrollo de proyectos científicos, bajo el títuño: Programa de Jóvenes Investigadores (PII). Para evaluar su efecto en términos de desarrollo de proyectos de investigación y participación científica en estudiantes del Nivel Medio Superior (NMS), se aplicó dicho programa a un grupo de 10 estudiantes de bachillerato de la Universidad Autónoma de Nuevo León. Los proyectos desarrollados por los estudiantes participantes durante la aplicación del programa se enfocaron en temas de biotecnología y biología molecular, generando proyectos de su propia autoría enfocados en resolver problemas sociales. Se pudo observar que se cumplieron los objetivos del programa propuesto y que los estudiantes se motivaron a decidirse por carreras de contenido científica e involucrarse en la comunicación a través de actividades de divulgación científica.

PALABRAS CLAVE: Bachillerato - Motivación - Método - Enseñanza Científico - Programa.
ABSTRACT

The economic growth and international competitiveness of a country are closely related to its capacity for scientific and technological development (Méndez, 2000). Mexico is a country where the little investment in science and a low index in the academic level of the population, cause a lack of motivation among young people to decide on scientific and engineering careers and to dedicate themselves to the scientific and technological development of the country (Hernández, 2018). Despite the efforts shown by Conacyt and private institutions to carry out activities focused on the scientific motivation of high school students; few programs focus on the training of young researchers. This paper presents the development of an extracurricular program at the baccalaureate level whose objective is to encourage in high school students an interest in research and development of scientific projects, under the title: Young Researchers Program (PII). To evaluate its effect in terms of the development of research projects and scientific participation at high school level, this program was applied to a group of 10 high school students from the Universidad Autónoma de Nuevo León. The projects developed by participating students during the application of the program focused on biotechnology and molecular biology issues, generating projects of their own authorship focused on solving social problems. It was observed that the objectives of the proposed program were met and that students were motivated to decide on careers with scientific content and to get involved in communication through scientific outreach activities.

KEYWORDS: High School - Motivation - Method - Teaching - Scientist - Program.

DOI: $10.29105 /$ pu8.15-7 
E n México existe un importante rezago educativo a pesar de los múltiples esfuerzos que en los últimos años se han hecho en diferentes instancias educativas y gubernamentales para mejorar la situación. El alto apoyo para el desarrollo industrial y económico centrado en la técnica, ha dejado claramente en desventaja los recursos que se destinan para la investigación y la educación (Contreras, 2017).

Este desbalance ha repercutido en la población, convirtiéndonos en un país en donde la sociedad no se encuentra bien informada sobre los desarrollos tecnológicos existentes en el mundo, generándose además una cultura de rechazo hacia aquellas personas que deciden guiarse por carreras o programas científicos. Es normal sorprendernos por tecnologías que provengan de otros países y que califiquemos, desde nuestro punto de vista, como de primer nivel, impidiéndonos prestar atención a la poca tecnología también, de calidad, que se ha logrado desarrollar en nuestro país con el esfuerzo y dedicación de sólo unos pocos.

Actualmente estamos en contacto constante con la información a través de Internet, aunque, se sabe, no toda la que circula por ella es necesariamente verídica, requiriéndose tener una mente analítica para ahondar más en la información y llegar a la verdad.

Lamentablemente, los jóvenes son los usuarios más afectados por la desinformación hoy en día, generando confusión entre ellos impidiéndoles discernir lo que es verídico de lo que no lo es y que perciban con claridad el alcance que puede lograr el país si prioriza el desarrollo de la ciencia.
El objetivo de esta investigación se centra en la elaboración de un programa interdisciplinario enfocado principalmente en elevar el grado de interés de los jóvenes en la ciencia y en aumentar su participación científica a través del desarrollo de proyectos, específicamente de aquellos jóvenes que se encuentran cursando el Nivel Medio Superior. A través de este programa se espera poder introducir a los participantes a temas de ciencia antes que se decidan por una carrera profesional y motivarlos por la investigación y la innovación tecnológica para la generación de nuevos conocimientos científicos de interés social 0 industrial.

De acuerdo con Méndez (2000), para que un país logre obtener un gran crecimiento económico y aumente su competitividad internacional, es necesario su desarrollo científico y tecnológico. Gran parte de los países cuyos habitantes gozan de una alta calidad de vida y que son considerados de economía estable, apuestan al desarrollo de la ciencia y la tecnología con la finalidad de comercializar sus bienes y servicios. Esto a su vez logra mejorar las condiciones socioeconó- micas de sus habitantes y mantener su bienestar. Según la revista Forbes (2008), aunque México ha intentado establecerse como un país modelo en Latinoamérica, todavía se encuentra muy lejos de convertirse en un país competitivo en el desarrollo científico.

En la actualidad, la calidad de vida de muchos de los habitantes de nuestro país se encuentra comprometida debido a la falta de servicios básicos como la salud, el acceso a la seguridad social, la calidad y espacios en la vivienda; así como por la pobreza extrema y la falta de educación (Sedesol, 2011). Aunque los gobiernos han tratado de mitigar esta situación en la que se encuentra más del $60 \%$ de la población mexicana, no se presta atención al potencial con el que cuenta el desarrollo tecnológico para reducir esta condición. De acuerdo con Aldana (2012), el déficit de inversión en ciencia y tecnología y la ausencia de una cultura científica entre la población, son factores que frenan el desarrollo científico de nuestro país.

De acuerdo con el diario El Universal (2008), países como Estados Unidos y China invierten más del $1 \%$ de su

\section{De acuerdo con Méndez (2000), para que un país} logre obtener un gran crecimiento económico y aumente su competitividad internacional, es necesario su desarrollo científico y tecnológico 
PIB anual al desarrollo científico y tecnológico; mientras que en México solamente se invierte el $0.33 \%$ del PIB anual. Esto se debe principalmente a la falta de cultura científica que se vive en nuestro país y representa la realidad en la que se encuentra la ciencia en México, representando un reto para las organizaciones gubernamentales que administran estos recursos y la distribución de los mismos (Aldana, 2012). Tal es el caso del Conacyt, que con la finalidad de impulsar programas de formación científica en los jóvenes, cuenta con programas de becas para estudios de posgrado y convocatorias para la realización de actividades de divulgación científica (Conacyt, 2018).

Otros centros, institutos y universidades han ofrecido programas que motivan a los jóvenes al estudio de las ciencias, sustentados por la idea de que el desarrollo tecnológico y científico es aquel que traerá crecimiento y estabilidad económica a nuestro país. No obstante, gran parte de estos programas se centran en estudiantes de licenciatura y posgrado, dejando de lado el potencial de los estudiantes de bachillerato.

Aunque existen actividades enfocadas a la motivación científica de los jóvenes de bachillerato como la Feria Nacional de Ciencias e Ingenierías organizada por el Gobierno de la República a través del Conacyt, son pocos los programas mexicanos que se enfocan en la formación de jóvenes investigadores. A pesar de los esfuerzos mostrados por el Conacyt o instituciones privadas en ofrecer oportunidades de apoyo a proyectos de investigación para jóvenes, o becas de estudios de posgrado, aún existe mucha indiferen-

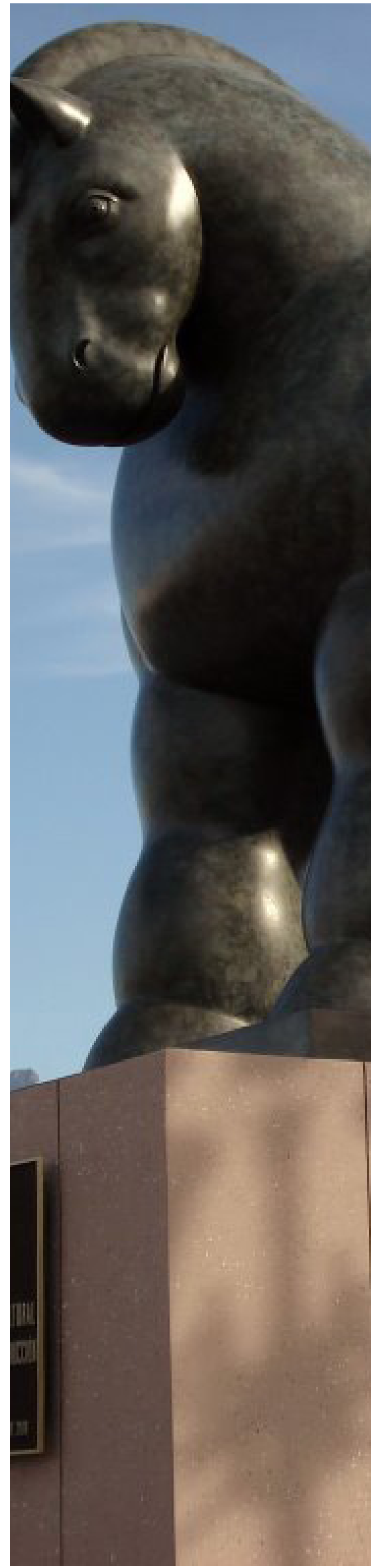

cia por parte de los jóvenes de ser parte de dichos programas (Campanario, 2000).

De acuerdo al diario El Financiero (2018), en México, a más del $80 \%$ de los jóvenes que se encuentran cursando el bachillerato no le interesa estudiar alguna carrera enfocada a la ciencia 0 a la ingeniería, siendo estas incluso de las mejor pagadas. Esto se debe principalmente a la falta de motivación que existe hacia estos jóvenes por parte de sus padres y de sus profesores, incluso pueden llegar a desmotivarse si el profesor tampoco demuestra interés en su propia clase (Contreras, 2007).

El objetivo del programa desarrollado en esta investigación es fomentar en los estudiantes la investigación científica y el desarrollo de proyectos científicos. Se busca también ofrecerles la oportunidad de poner en práctica los conocimientos que han adquirido durante su formación académica a través del desarrollo y ejecución de proyectos interdisciplinarios de su propia autoría. Todo lo anterior a través de su participación activa en la búsqueda de información, el diseño experimental, la práctica en el laboratorio, el análisis de la información, el uso de las TIC y el desarrollo de habilidades de comunicación científica. Finalmente, el programa propuesto integra las diferentes características de la corriente pedagógica constructivista descrita por Vygotsky en los años 60.

\section{MÉTODO}

Con la finalidad de aumentar el interés de los jóvenes que están cursando el Nivel Medio Superior en temas de ciencias e incrementar su participación científica, se desarrolló un pro- 
grama de investigación científica en la modalidad extracurricular titulado: Programa de Jóvenes Investigadores (PJI) y se evaluó el comportamiento de los estudiantes en cuanto a su participación científica y motivación por medio de un estudio de tipo exploratorio.

Este programa se aplicó a un grupo de 10 estudiantes de bachillerato de la Universidad Autónoma de Nuevo León, a los que previamente se les dio un curso intensivo de introducción a la Biotecnología y Biología Sintética durante una semana en 60 horas. Por tal razón, los proyectos desarrollados durante la aplicación del programa se enfocaron en temas de Biotecnología y Biología Molecular.

En el diseño del programa se incluyeron 6 rubros 0 ejes que regían las actividades y objetivos del mismo, esto con la finalidad de que los estudiantes participantes desarrollaran las habilidades científicas necesarias para incrementar su interés por las ciencias y su participación científica:

- Investigación Científica

- Trabajo Experimental

- Interdisciplinariedad

- Responsabilidad

- Bioseguridad

- Comunicación Científica

Investigación Científica: A través de la búsqueda de información científica y el análisis de contenidos en textos científicos, se les solicitó a los participantes del PJI diseñar proyectos con una aplicación directa a la resolución de problemas de la actualidad. De la misma manera, se les solicitó que, basándose en su investigación, desarrollarán una estrategia experimental que les permitiera llevar a cabo su proyecto en el laboratorio.
Trabajo Experimental: Una vez definida la estrategia experimental en el rubro de la Investigación Científica, con la asesoría del instructor y siguiendo las medidas de seguridad del laboratorio, se llevó a cabo el desarrollo del proyecto propuesto por el estudiante de forma experimental. Todo esto siguiendo los protocolos establecidos por los propios estudiantes. Se les pidió a los estudiantes documentar todo lo realizado durante la fase de experimentación a través de una bitácora personal.

Interdisciplinariedad: Tomando en cuenta que un proyecto científico no concierne a una sola área del saber, se propuso que de acuerdo al proyecto desarrollado por cada estudiante, integrará otros aspectos dentro del área de las ciencias experimentales, ciencias exactas, ciencias de la comunicación y ciencias sociales. Para esto, dependiendo del proyecto, se le planteó al estudiante utilizar ecuaciones diferenciales sobre las cuales basa la producción de ciertos elementos clave en el proyecto, desarrollar un prototi-

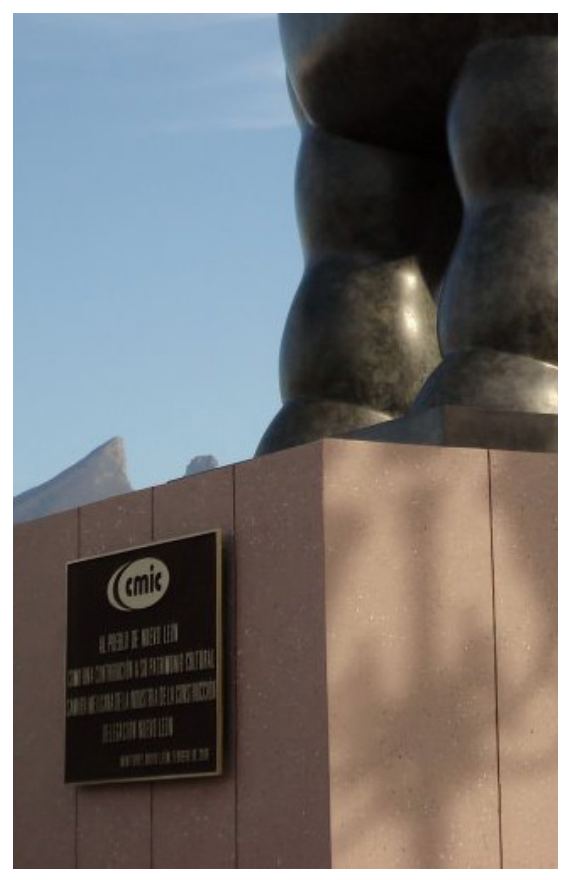

po de su proyecto a través de la impresión 3D y generar diseños gráficos de póster y presentaciones digitales para la exposición de los resultados de sus proyectos.

\section{Responsabilidad Social y Divulgación}

Científica: Se planteó al estudiante realizar actividades de divulgación científica con la finalidad de concientizar a la población de la importancia de la ciencia y cómo esta puede resolver problemas sociales. También se le propuso evaluar el impacto social que podría tener el desarrollo de su proyecto. Todo esto por medio de pláticas en algunos planteles educativos, conferencias, talleres o dinámicas que los estudiantes realizaron a través de su creatividad, integrando aspectos artísticos y científicos para la divulgación de la ciencia.

Ética y Bioseguridad: Se estableció un espacio reflexivo con los estudiantes para concientizarlos sobre las implicaciones éticas y de seguridad que conlleva la realización de su proyecto de investigación científica. También se

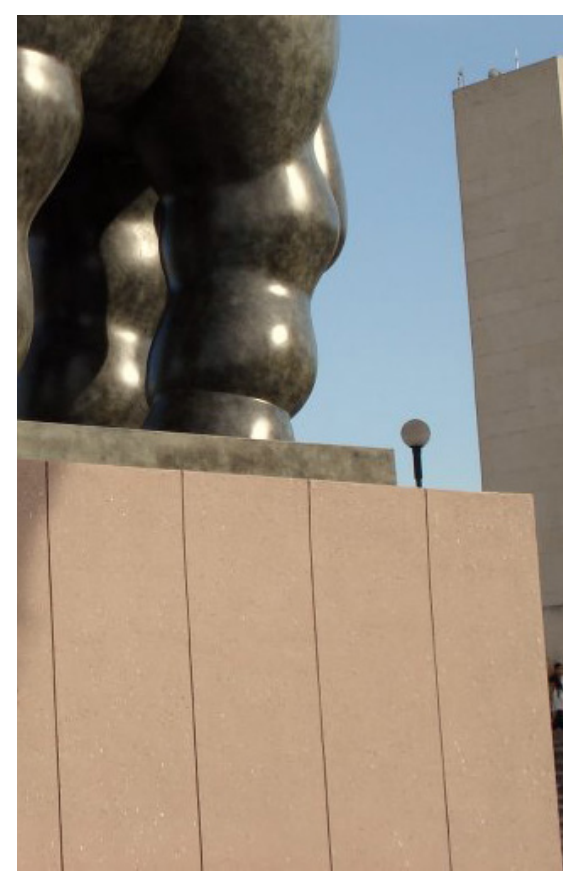


propuso la realización del análisis de riesgo y las implicaciones de seguridad tanto dentro como fuera del laboratorio. Todo lo anterior por medio de debates y discusiones.

Comunicación Científica: Se motivó a los estudiantes a participar en la exposición de sus proyectos y sus resultados obtenidos en diferentes congresos, simposios, foros, ferias de ciencias, debates y talleres de investigación en diferentes ciudades y universidades. Esto con la finalidad de que vivan la experiencia de exponer frente al público y desarrollen sus habilidades de comunicación oral y escrita. De la misma manera, se propuso a los estudiantes escribir sus proyectos a manera de artículo científico como ejercicio de comunicación de la ciencia.

Se observó el comportamiento de los estudiantes a lo largo del tiempo de ejecución del programa y se documentaron las actividades realizadas.

\section{RESULTADOS}

Dentro de los rubros de Investigación Científica, Trabajo Experimental e Interdisciplinariedad, los estudiantes participantes del programa desarrollaron 7 proyectos presentados en la Tabla 1, todos enfocados en el área de la biotecnología y la biología molecular. Los estudiantes trabajaron durante distintas temporadas durante 5 semestres desde finales del año 2017 hasta el semestre en curso (Agosto-Diciembre 2019) en el diseño de dichos proyectos a través de la investigación científica, el diseño de experimentos y el trabajo en el laboratorio, obteniendo diversos resultados que fueron presentados en algunos eventos científcos para jóvenes tales como ferias de ciencias y congresos, entre otros.

De igual manera, presentaron una integración de sus conocimientos en las áreas de matemáticas, física, química, biología y las TIC tanto para la planeación como para la ejecución de

\section{Tabla 1. Proyectos elaborados por los estudiantes participantes en el programa de investigación científica}

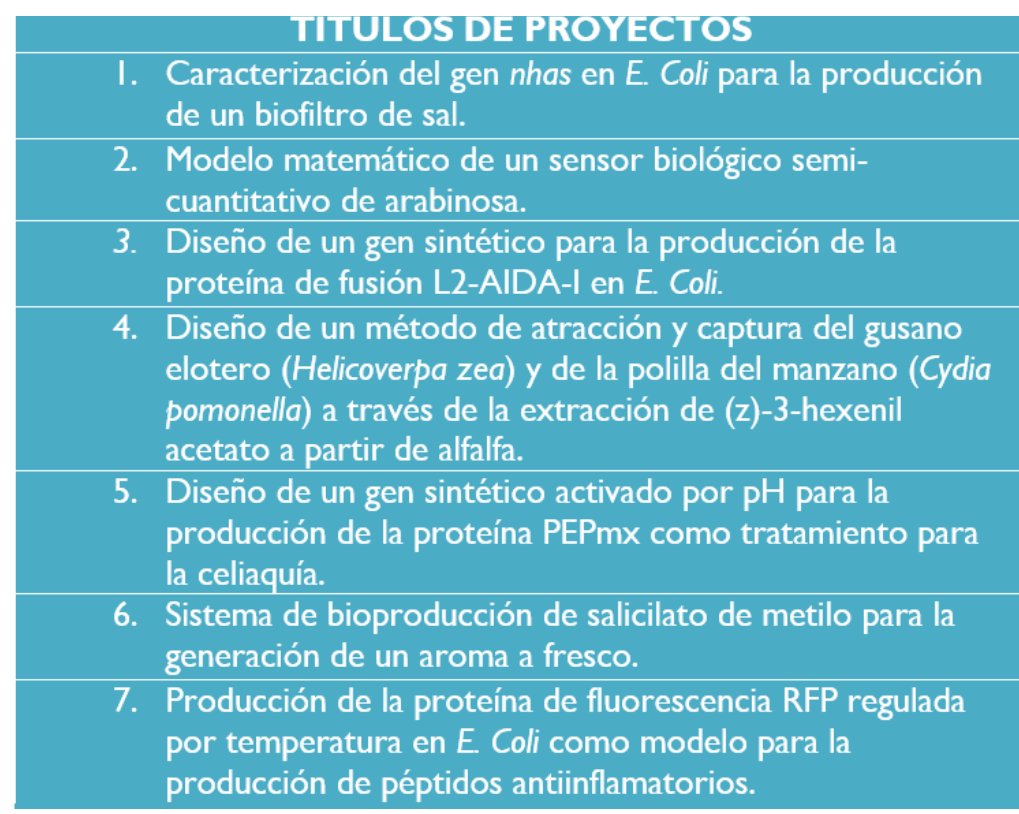

sus proyectos. En el caso del rubro de Comunicación Científica, los estudiantes participaron en diversas ferias de ciencias presentando sus proyectos a través de carteles científicos y de divulgación, así como ponencias en simposios y congresos de nivel licenciatura y posgrado a nivel nacional, obteniendo resultados sobresalientes. En la Tabla 2 se muestran las participaciones del grupo de estudiantes y los resultados obtenidos.

Dentro del rubro de Responsabilidad Social y Divulgación Científica, los estudiantes tuvieron la oportunidad de asistir a un taller en el laboratorio de Ingeniería Biológica del Instituto Tecnológico de California (Caltech) en la ciudad de Pasadena, California en el mes de julio del 2018. Asimismo, participaron en la realización de diversos talleres de divulgación científica para jóvenes tanto de nivel de educación secundaria como de bachillerato durante los meses de enero, junio y julio del 2018 y enero, febrero, abril y junio del 2019.

Cabe mencionar que para el rubro de Ética y Bioseguridad, los estudiantes realizaron análisis de riesgos biológicos de los diferentes proyectos desarrollados antes de su experimentación, así como una preparación otorgada por el personal de laboratorio de la preparatoria en el uso de materiales, reactivos, equipos e instrumentación de laboratorio.

\section{CONCLUSIONES Y PROPUESTAS DE ACCIÓN Y TRANSFORMACIÓN}

Los estudiantes participantes en el programa generaron proyectos de su propia autoría, enfocados en resolver problemas sociales dentro de las áreas de la biotecnología y la biología 
Tabla 2. Participación de los estudiantes en diversas actividades científicas en las que presentaron diversos proyectos desarrollados y los resultados obtenidos

\begin{tabular}{|c|c|c|c|}
\hline $\begin{array}{c}\text { NOMBRE } \\
\text { DEL EVENTO }\end{array}$ & $\begin{array}{c}\text { TIPO DE } \\
\text { PARTICIPACIÓN }\end{array}$ & FECHA & RESULTADO \\
\hline $\begin{array}{c}\text { 2do Foro de } \\
\text { Biología Sintética en } \\
\text { México } \\
\end{array}$ & $\begin{array}{c}\text { Presentación de cartel } \\
\text { científico }\end{array}$ & Diciembre 2017 & Mención honorífica \\
\hline $\begin{array}{c}\text { V Congreso } \\
\text { Internacional de } \\
\text { Biotecnología } \\
\text { Genómica, } \\
\text { Genobiotec I8 } \\
\end{array}$ & Asistente & Junio 2018 & $\begin{array}{l}\text { Participación como } \\
\text { oyente }\end{array}$ \\
\hline $\begin{array}{c}\text { Congreso Nacional } \\
\text { de Ciencias iGEM } \\
2018 \\
\end{array}$ & Asistente & Julio 2018 & $\begin{array}{c}\text { Participación como } \\
\text { oyente }\end{array}$ \\
\hline $\begin{array}{c}\text { Feria Nacional de } \\
\text { Ciencias e } \\
\text { Ingenierías 2018 }\end{array}$ & $\begin{array}{l}\text { Presentación de cartel } \\
\text { científico y proyecto } \\
\text { de investigación }\end{array}$ & Septiembre 2018 & $\begin{array}{l}\text { Ier, 2do y 3er lugar } \\
\text { en la categoría de } \\
\text { Salud y Medicina }\end{array}$ \\
\hline ExpoCiencias 2018 & $\begin{array}{c}\text { Presentación de cartel } \\
\text { científico y proyecto } \\
\text { de investigación }\end{array}$ & Septiembre 2018 & $\begin{array}{l}\text { Participación como } \\
\text { expositor }\end{array}$ \\
\hline $\begin{array}{l}\text { Feria Nacional de } \\
\text { Ciencias e } \\
\text { Ingenierías 2018 - } \\
\text { Fase Nacional }\end{array}$ & $\begin{array}{l}\text { Presentación de cartel } \\
\text { científico y proyecto } \\
\text { de investigación }\end{array}$ & Noviembre 2018 & $\begin{array}{c}\text { Representante del } \\
\text { estado en la } \\
\text { delegación de } \\
\text { Nuevo León }\end{array}$ \\
\hline $\begin{array}{l}\text { 8vo Congreso } \\
\text { Internacional de } \\
\text { Biotecnología } \\
\text { Quorum }\end{array}$ & $\begin{array}{l}\text { Presentación de cartel } \\
\text { científico }\end{array}$ & Marzo 2019 & $\begin{array}{c}\text { 2do lugar en } \\
\text { presentación de } \\
\text { carteles científicos }\end{array}$ \\
\hline $\begin{array}{c}\text { Journey to } \\
\text { Innovation del } \\
\text { Tecnológico de } \\
\text { Monterrey }\end{array}$ & $\begin{array}{c}\text { Presentación de cartel } \\
\text { científico }\end{array}$ & Abril 2019 & $\begin{array}{l}\text { Participación como } \\
\text { expositor }\end{array}$ \\
\hline $\begin{array}{l}\text { Exposición de } \\
\text { Cartel en Expo } \\
\text { Científica de CTR }\end{array}$ & $\begin{array}{c}\text { Presentación de cartel } \\
\text { científico }\end{array}$ & Мауо 2019 & $\begin{array}{l}\text { Participación como } \\
\text { expositor }\end{array}$ \\
\hline
\end{tabular}




\section{Los estudiantes participantes en el programa}

\section{generaron proyectos de su propia autoría,}

\section{enfocados en resolver problemas sociales}

dentro de las áreas de la biotecnología

y la biología molecular

molecular. De esta misma forma integraron tanto conocimientos previos como aquellos obtenidos en clase, en el desarrollo y en la ejecución de sus proyectos, realizando actividades de laboratorio e incorporando elementos de otras disciplinas como matemáticas, química, física, artes y ciencias sociales.

Los participantes también realizaron actividades de divulgación científica a través de la realización de talleres y pláticas en algunas escuelas de la ciudad, demostrando sus habilidades de comunicación y de socialización. Esto les permitió tener un mejor acercamiento a las necesidades sociales que llevaron a una mejor motivación para la mejora de la calidad de vida de la población.

Finalmente, tuvieron la oportunidad de participar en diversas conferencias, simposios, presentación de carteles científicos, congresos, talleres y ferias estatales y nacionales, presentando sus trabajos y obteniendo muy buenos resultados debido a su buen desempeño. Del grupo de estudiantes participantes a inicios del desarrollo del programa, todos decidieron estudiar carreras relacionadas con las ciencias experimentales y las ingenierías.

Se puede concluir también que el programa motivó a los estudiantes a decidirse por carreras científicas e involucrarse en la comunicación científica a través de actividades de divulgación. Se propone aplicar el Programa de Jóvenes Investigadores en más preparatorias con la finalidad de ampliar la investigación e impactar en los estudiantes del NMS sobre su decisión y motivación en el estudio de las ciencias experimentales.

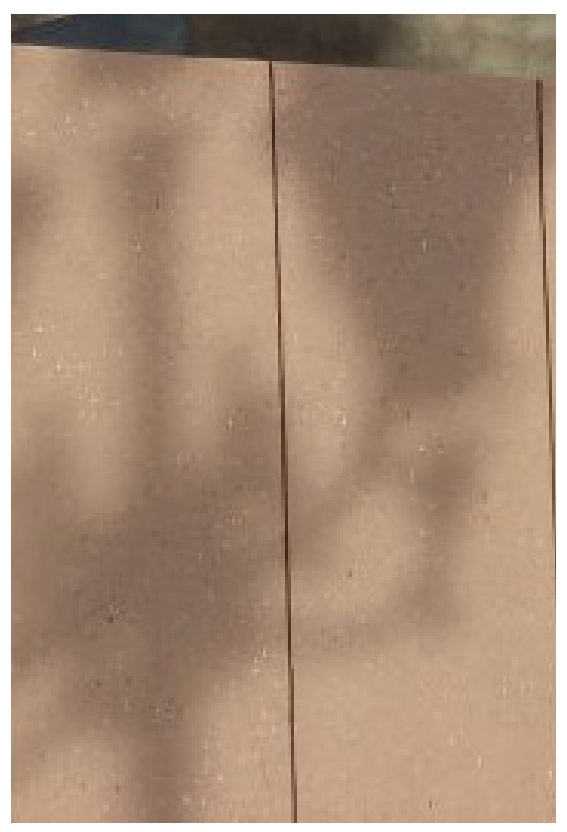

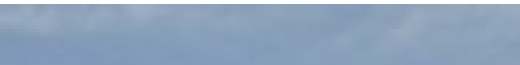
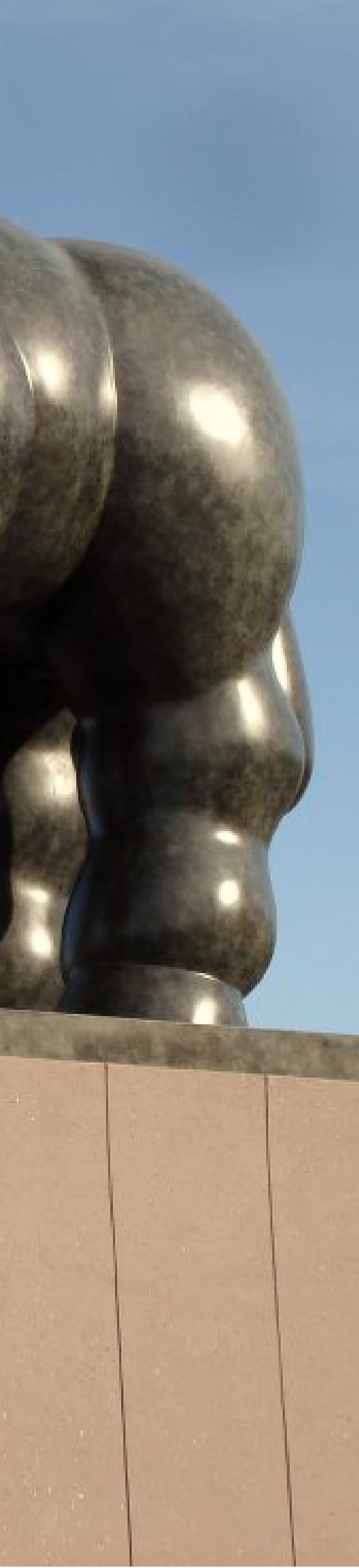


\section{REFERENCIAS}

Aldana, M. (2012). ¿Qué le falta a la ciencia en México? Temas UNAM. No. 69: 26-30, enero-marzo de 2012.

Campanario, J. M Y Otero, J.C.(2000). Más allá de las ideas previas como dificultades de aprendizaje: Las pautas de pensamiento, las concepciones epistemológicas y las estrategias metacognitivas de los alumnos de ciencias. Enseñanza de las Ciencias, vol. 18, núm. 2, pp.155-169.

Contreras, R. (2017, Marzo, 25). Recorte en ciencia y tecnología. 25 de Marzo de 2017. Excélsior. Recuperado de http://www.excelsior.com.mx/opinion/raul-contreras-bustamante/2017/03/25/1154002.

Convocatorias y resultados Conacyt. (2019,10 de Agosto). Obtenido de: https:// www.conacyt.gob.mx/index.php/el-conacyt/convocatorias-y-resultados-conacyt Fortes, Mauricio. (2001). Educación y Retos Sociales de los Avances de la Ciencia. ITESO. México. Revista Electrónica Sintética. numero 18, enero junio 2001, pp. 69-76.

Hernández, L. (28 de Septiembre de 2018). Falta de estudiantes de Ciencias y Tecnología en México preocupa a empleadores. El Financiero. Recuperado de: https://www.elfinanciero.com.mx/economia/falta-de-estudiantes-de-ciencias-y-tecnologia-en-mexico-preocupa-a-empleadores

Méndez, E. (2000). El desarrollo de la ciencia. Un enfoque epistemológico. Espacio Abierto, vol. 9, núm. 4, octubre-diciembre, 2000, pp. 505-534

Sedesol. (2011). Medición de la pobreza. Servicios básicos de la vivienda. Publicación informativa de la Subsecretaría de Prospectiva, Planeación y Evaluación. Núm. 7, Julio de 2011.

Vygotsky, L. (1962). Thought and language. Cambridge, MA: MIT Press.

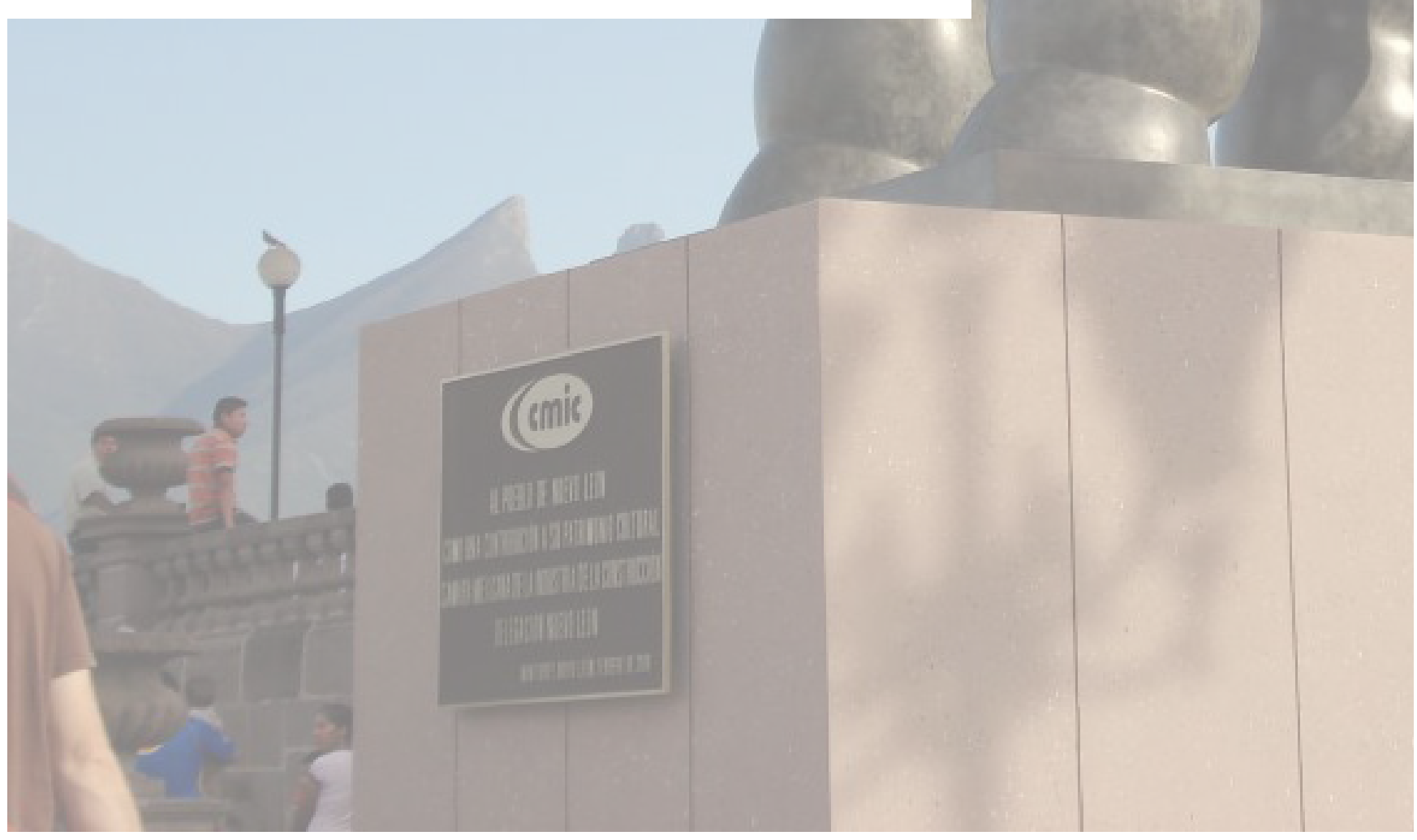




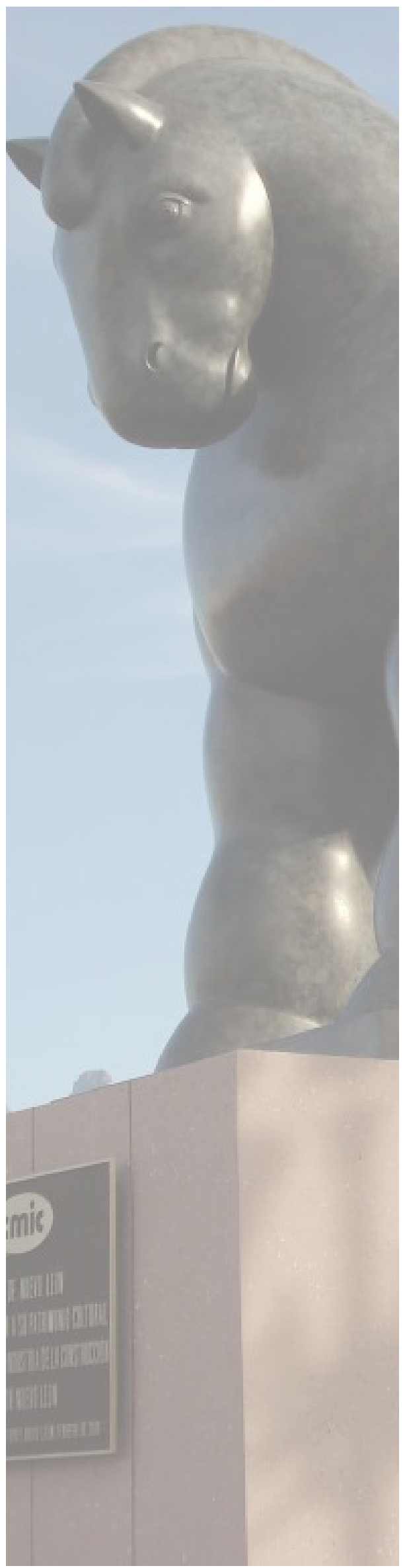

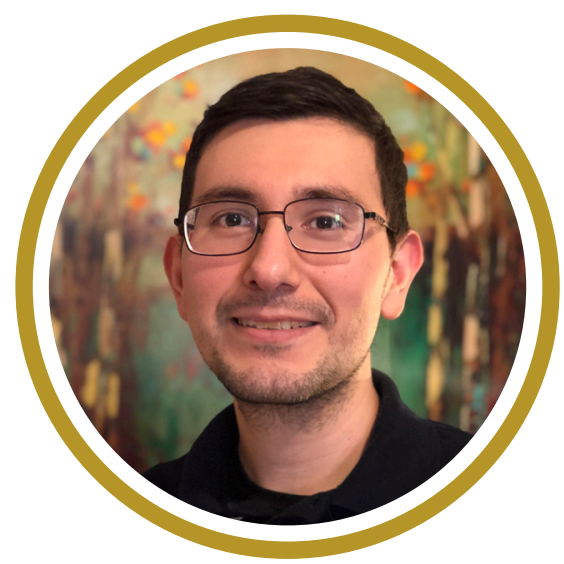

\section{Heber Miguel \\ Torres Cordero}

Licenciado en Biotecnología Genómica por la UANL y Maestro en Ciencias en Biomedicina Molecular por el CINVESTAV-IPN. Doctorando en Filosofía con Especialidad en Comunicación e Innovación Educativa, especializado en divulgación y educación científica a nivel bachillerato. Profesor del NMS en la UANL con 8 años de experiencia y creador de contenidos para la educación científica en jóvenes dentro de la SI-UANL y Scintia.

\section{Correo Electrónico:}

hebersco@hotmail.com

Fecha de Recepción: 15/03/20

Fecha de Aprobación: 15/08/20 